\title{
Letter to the Editor: Cyclic Nucleotide Phosphodiesterase Activity of Human Liver: Is There an Altered Form in Reye's Syndrome?
}

\author{
ROBERT A. MITCHELL
}

Department of Biochemistry, Wayne State University School of Medicine, Detroit, Michigan, USA

In a recent article, Dr. Kang (1) reported differences in the cAMP phosphodiesterase activity in liver specimens of Reye's syndrome patients compared to a control group. In particular, she claimed to find an increase (approximately 3 -fold) in the $K_{n}$ for the low affinity cAMP form. From this and related kinetic data, she suggested that there was "the distinct possibility that hormone responsiveness may be abnormal in this condition and that a premorbid phenotype may be characteristic of children who, at least, succumb to Reye's syndrome." The validity of this conclusion requires comment.

For the past year, work in my laboratory with the support of the Reye's Syndrome Study Center of the Children's Hospital of Michigan, has been directed at the study of enzyme alterations in the liver of Reye's syndrome patients. In agreement with the published work of others, we have observed decreases in the activity of some enzymes, particularly mitochondrial enzymes. It is highly unlikely that each of these alterations is a consequence of a separate marginal type of inborn error. It is more likely that the decreases in activity are mediated in some manner by a regulatory process, as yet poorly understood. Dr. Kang's suggestion of a disturbance in cAMP chemistry in Reye's syndrome thus requires careful scrutiny.

There are two aspects of her work which cause concern. First, the method used to calculate the kinetic constants was not referenced. If by, "the double reciprocal plot method", Dr. Kang means that values were calculated from the two limbs of a $1 / \mathrm{v} v \mathrm{vs}$. $1 / \mathrm{S}$ plot, this procedure is not sufficient. This method yields only first estimates which may be far from the true values (2). This is not a trivial objection, because alterations in the relative amounts of two such enzymes (i.e., alterations in the observed $V_{m}$ values) would in general alter the shape of the plots. Such change might be erroneously interpreted as being due to alteration in $\mathrm{K}_{\mathrm{m}}$ values, unless a proper analysis is carried out. The correct procedure for the analysis of this type of data is an iterative one, because the contribution of the enzyme predominant in one limb to the activity observed in the other limb needs to be repeatedly assessed (3). If this was not done, then the displayed values are erroneous, and it is not possible to know if any changes observed are due to alterations in $\mathrm{K}_{\mathrm{m}}, \mathrm{V}_{\mathrm{m}}$, or both.

A second objection is that the illustrative data presented in Figure 4 does not provide convincing evidence for the presence of multiple types of diesterase activity, when compared say, with the results from similar types of experiment with rat brain (4).

To better examine Dr. Kang's data for human liver, and Drs. Thompson's and Appleman's data for rat brain, Figure 4 of the former and Figure 1 of the latter were photographically enlarged, and the coordinates of points ( $v v s$. S plots) were measured using an electronic digitalizer. Scale factors were calculated from coordinates of points taken along the $x$ and the $y$ axes. The $v$ and $s$ values so obtained were analyzed using a computer program written for the purpose of analyzing this type of kinetic data (5). For $n$ sets of coordinates, there are $n-3$ ways of grouping the points into high and low substrate blocks (it requires at least 2 points to draw a straight line). In general, it is to be expected that the best resolution will be obtained when the low and high substrate blocks are selected with the dividing point close to the break in the reciprocal plot, although in practice it is simple to test all possible dividing points. In this way, a numoer of different sets of estimates can be obtained, for a cluster of groupings with dividing points close to the break in the line.

Analysis of the human liver data by this means failed to yield any realistic set of kinetic constants, e.g., negative $\mathrm{K}_{\mathrm{m}}$ values were found, regardless which of the four dividing points was used. In contrast, for the brain enzyme data, realistic values were obtained for all estimates of the low affinity cAMP enzyme, but in particular, a cluster of four of the twelve possible groupings tested yielded similar estimates for all four of the kinetic constants $\left(\mathrm{K}_{\mathrm{m} \text { (high) }}=45-52 \mu \mathrm{M} ; \mathrm{V}_{\mathrm{m} \text { (high) }}=213-220\right.$ units; $\mathrm{K}_{\text {m(low) }}=1.4-2.9$ $\mu \mathrm{M} ; \mathrm{V}_{\text {m(low) }}=8.6-14.6$ units). The values could probably be refined upon further analysis, but this was not attempted.

There are several possibilities to explain why the human liver experiments differed from those with rat brain. Human liver may lack such forms, or a form may have been inactivated before analysis. The enzymes may exist, but their kinetic constants may be such as to render the forms incapable of kinetic resolution (2). The initial rate studies of Dr. Kang may not have been sufficiently accurate to delineate the forms.

In summary, the conclusion by Dr. Kang that there is an altered type of phosphodiesterase activity in Reye's syndrome cases compared to the control group is unwarranted, because the method of analysis used to treat the data was not appropriate for this type of purpose. In addition, when the correct type of analysis was applied to the illustrative data presented in Figure 4 of her paper, no feasible sets of kinetic constants could be calculated, i.e., the data were not indicative of two forms of enzyme being present.

\section{REFERENCES AND NOTES}

I. Kang, E. S.: Cyclic nucleotide phosphodiesterase activities of human liver: Comparison between control and Reye's syndrome samples. Pediatr. Res., 12: 761 (1978).

2. Segel, I. H.: In Enzyme Kinetics, p. 64-71 (John Wiley \& Sons, New York, 1975).

3. Spears, G., Sneyd, J. G. T., and Loten, E. G.: A method for deriving kinetic constants for two enzymes acting on the same substrate. Biochem. J., 125: 1149 (1971).

4. Thompson, W. J., and Appleman, M. M.: Multiple cyclic nucleotide phosphodiesterase activities from rat brain. Biochemistry, 10:311 (1971).

5. Russo, J. A., Lamos, C. M., and Mitchell, R. A.: Occurrence of an uncouplerresistant intermediate type of phosphate water oxygen exchange reaction catalyzed by heart submitochondrial particles. Biochemistry, 17: 473 (1978).

6. Requests for reprints should be addressed to: Dr. Robert A. Mitchell. Department of Biochemistry, Wayne State University, Scott Hall, 540 E. Canfield, Detroit. MI. 48201, USA. 